\title{
A direct comparison between a MEMS deformable mirror and a liquid crystal spatial light modulator in signal-based wavefront sensing
}

\author{
A. R. Jewel \\ md.atikur.jewel@ucdconnect.ie \\ V. Akondi
}

B. Vohnsen
Advanced Optical Imaging Group, School of Physics, University College Dublin, Dublin 4, Ireland

Advanced Optical Imaging Group, School of Physics, University College Dublin, Dublin 4, Ireland

\begin{abstract}
Aberrations degrade the performance of optical systems in terms of resolution and signal-to-noise ratio. This work explores the feasibility of a signal-based wavefront sensor, which employs a search algorithm to estimate Zernike coefficients of given aberrations. The search algorithm was supported by Gaussian interpolation. The performance of two different reflective wavefront correctors, a deformable mirror and a spatial light modulator in signal-based wavefront sensing, was compared under identical conditions. The aberrations were introduced by using another identical high resolution reflecting spatial light modulator. The performance was quantified using the Strehl ratio, which was estimated from simultaneously acquired Hartmann-Shack measurements of Zernike coefficients. We find that the spatial light modulator can be a good alternative to the deformable mirror in terms of dynamic range and sensitivity, when speed is not a limiting factor. Distinct advantages of the spatial light modulator are high number of pixels and a larger active area.
\end{abstract}

[DOI: http://dx.doi.org/10.2971/jeos.2013.13073]

Keywords: Wavefront sensing, adaptive optics, aberration compensation, deformable mirror, spatial light modulator

\section{I NTRODUCTION}

Conventional wide-field microscopes and confocal microscopes can produce images with a resolution down to sub-micrometer scale. Fluorescence based microscopy methods provide functional information of cellular processes and chemical specificity $[1,2]$. New methods of nanoscopy that combine optical and photophysical phenomena can resolve details of a specimen in tens-of-nanometer scale [3]. However, the specimen generally has inhomogeneous optical properties and exhibits a spatially varying refractive index and absorption that reduce the resolution and imaging efficiency $[4,5]$. In scanning fluorescence microscopy, the intensity of the fluorescent emission decreases due to aberrations of the excitation light. Using higher intensities to compensate this effect can cause photo-bleaching and photo-toxicity [6].

Aberrations vary with specimen and cannot be fully corrected in a static optical design. A dynamic wavefront correction is required with Adaptive Optics (AO). A typical AO system contains a Hartmann-Shack (HS) wavefront sensor (WFS) and a deformable mirror (DM) or a spatial light modulator (SLM) to sense and correct the wavefront aberrations [7].

The use of a HS WFS in microscopy is complicated by the fact that a suitable guide star may not be present and instead a confocal sensing technique has been proposed to limit the axial depth [8]. Moreover, the ability of the HS WFS in precisely estimating the wavefront shape depends on the number of detector pixels corresponding to a single sub-aperture, centroiding errors and noise mechanisms $[7,9,10]$.

To circumvent this limitation, and as microscopy ultimately relies on quality parameters of the signal and images, wavefront sensor-less AO systems based on the maximization of a detector signal or sequential optimization of the adaptive correction elements has been introduced [11]. Genetic algorithms were employed to maximize second-harmonic signals and two-photon fluorescence, laser light coupling into an optical fiber and in reflection microscopy [12]-[16]. Hill climbing algorithm [17], stochastic parallel gradient descent [18] and modal-based wavefront sensing methods were implemented $[11,19]$.

In this paper, a wavefront sensor-less approach is described based on sequential maximization of the detected power, $P$, using a photodetector placed behind a pinhole, which acts like a spatial filter at the focal plane. A DM and an SLM were successively employed as adaptive elements to correct aberrations under identical conditions and their performance was compared. A commercial HS WFS was used as a standard in both systems to evaluate the performance of the developed signal-based AO system.

\section{METHOD}

Wavefront aberrations, $\phi$ can be represented in terms of Zernike polynomials as follows:

$$
\phi(r, \theta)=\sum_{i=1}^{i=\infty} a_{i} Z_{i}(r, \theta)
$$

where $a_{i}$ is the coefficient of Zernike polynomial $Z_{i}(r, \theta)$. The indexing scheme used is explained in Appendix A. 


\subsection{Search algorithm}

Before starting the correction process, the pinhole was adjusted to the location of maximum signal to reduce large magnitude tilt aberration and defocus that could otherwise mask the aberrations. Subsequently, the algorithm first searches for a suitable value, $a_{4}^{\text {est }}$ (an estimate for defocus) by keeping $a_{i}=0, \forall i \neq 4$ until $P$ is maximized. Next, a search for the value of $a_{1}^{\text {est }}$ (an estimate for $a_{1}$ ) is made such that by applying the recently estimated wavefront, $\phi^{\text {est }}(r, \theta)=a_{4}^{\text {est }} Z_{4}(r, \theta)+a_{1}^{\text {est }} Z_{1}(r, \theta)$ the detected power is maximized further. The range and step size over which the search for $a_{i}^{e s t}$ is made is critical. This procedure is continued up to the fourteenth $(i=14)$ Zernike polynomial in our case but can be carried on further to achieve a maximum power, $P$. However, lower-order aberrations have a dominant effect on the point-spread function when focusing the light through a refractive-index mismatch medium in confocal microscopy [19]. After correction, the estimated wavefront is given by,

$$
\phi^{e s t}(r, \theta)=\sum_{i=1}^{i=14} a_{i}^{e s t} Z_{i}(r, \theta)
$$

The residual wavefront error can be written, $\phi^{\text {residual }}=\phi-\phi^{\text {est }}$. It can be corrected further by assuming that the new wavefront phase is $\phi=\phi^{\text {residual }}$ and the entire process can be repeated a number of times. Here, three loops were chosen providing satisfactory results. The Strehl ratio is being optimized which is the ultimate goal in most wavefront correcting systems. On average for both systems, after the first loop $\sim 70 \%$, after the second loop $\sim 85 \%$ and after the third loop $\sim 87 \%$ of the wavefront error is corrected. In general, the performance of the DM is slightly superior to that of the SLM. Performing a normal search algorithm in finer steps would lead to better correction but at the cost of time. Different algorithms were implemented by others for correcting unknown aberration and also to minimize the number of measurements [20, 21]. In this paper, three-point Gaussian interpolation was applied in order to reduce the number of steps. Considering that for three different values of the search parameter, $\alpha_{i}=x_{1}, x_{2}, x_{3}$; the corresponding measured power is $p_{i}=y_{1}, y_{2}, y_{3}$; such that $y_{2}$ is the maximum measured power during the search of a single Zernike coefficient, then the estimated Gaussian interpolated Zernike coefficient, $x_{0}$ would be given by,

$$
x_{0}=\frac{1}{2} \cdot \frac{x_{3}^{2}-x_{2}^{2}+D\left(x_{1}^{2}-x_{2}^{2}\right)}{x_{3}-x_{2}+D\left(x_{1}-x_{2}\right)},
$$

where

$$
D=\frac{\ln \left(y_{2}\right)-\ln \left(y_{3}\right)}{\ln \left(y_{1}\right)-\ln \left(y_{2}\right)} .
$$

During the correction of each Zernike term, the achieved maximum powers by normal search and Gaussian search are compared and the coefficient value corresponding to the higher power among these two is chosen for correction.

\subsection{Simulation of random aberrations}

The measured Zernike coefficients of the aberrations introduced by a C. elegans sample, extracted from the literature [22], were used to generate random aberrations of relevance in microscopy. The mean (M) and standard deviation (S) of the

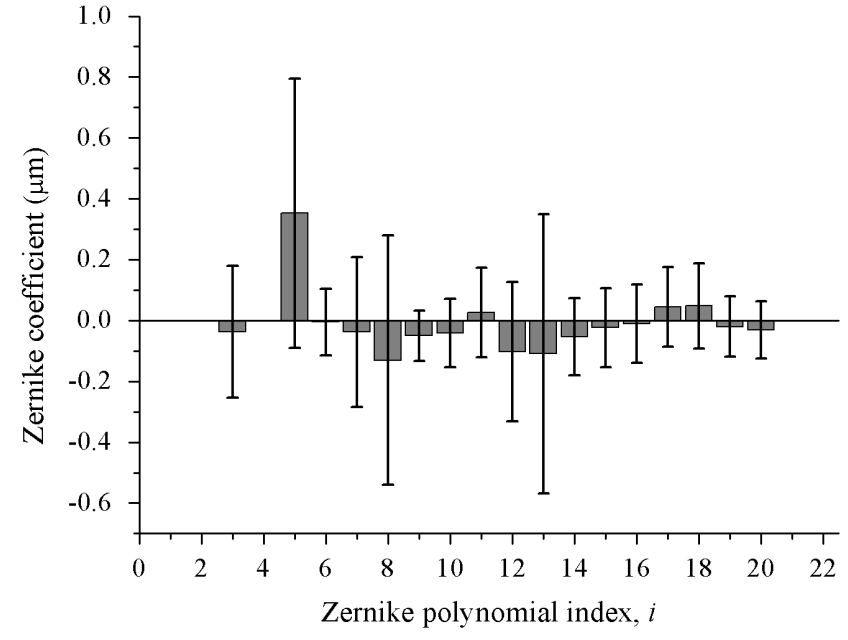

FIG. 1 Aberration statistics in C. elegans [22].

Zernike coefficients $\left(a_{i}\right)$ are shown in Figure 1. Using these statistics and Eq. (5), random Zernike coefficients were obtained from which random wavefronts were generated. As the tilt and defocus terms do not distort the shape of the focal spot, they were excluded in the simulation of random aberrations.

$$
a_{i}=R_{i} \cdot S_{i}+M_{i}
$$

where $\left(R_{i}\right)$ is an array of random numbers whose elements are normally distributed with mean 0 and variance 1 .

\subsection{Experimental setup}

Two different correcting elements, a DM and an SLM, were analyzed in this study using a single system as shown in Figure 2. A spatially-filtered He-Ne laser $(\lambda=632.8 \mathrm{~nm})$ with $3 \mathrm{~mm}$ beam diameter was used as light source. The MEMS based DM (Boston Micromachines, USA) with 140 actuators can be controlled by changing the voltage of each actuator (maximum $210 \mathrm{~V}$ ). These provide a maximum stroke of $3.5 \mu \mathrm{m}$ over a compact area and the clear aperture is $4.4 \mathrm{~mm}$. The actuator pitch of the DM is $400 \mu \mathrm{m}$. The frame rate of the DM is $>2 \mathrm{kHz}$. A liquid crystal based SLM 2 (LCOS-SLM, X10468-02 series from Hamamatsu, Japan) that has a pixel resolution of $800 \times 600$ and operates at $60 \mathrm{~Hz}$ was used as another corrective element. The pixel pitch of the SLM is $20 \mu \mathrm{m}$ and the fill factor is $95 \%$. The effective area of the SLM is $16 \mathrm{~mm} \times 12 \mathrm{~mm}$. This 8-bit SLM can modulate the phase $\sim 3 \pi$ at the chosen wavelength. A stop was used to select either the DM or $\mathrm{SLM}_{2}$ as the active corrective element in the system. The AO system also consists of a real time HS WFS $(39 \times 31$ lenslet array, Thorlabs, USA), that can operate at $15 \mathrm{~Hz}$. The focal length of the microlenses in the HS is $3.7 \mathrm{~mm}$ and the subaperture pitch is $150 \mu \mathrm{m}$. The aperture size of the HS is $5.95 \mathrm{~mm} \times 4.76 \mathrm{~mm}$ (maximum) and the sensitivity is $\lambda / 15$ root-mean-square (RMS) at the chosen wavelength. A reflecting SLM $_{1}$ (LCOS-SLM, X10468-02 series from Hamamatsu, Japan) was employed to introduce different low-order aberrations and aberrations generated using C. elegans statistics in the system. In order to create identical conditions and beam size restriction of the DM, only $150 \times 150$ pixels of the SLM were used. After the lens $f_{1}$ the beam diameter was $15 \mathrm{~mm}$. Lenses $f_{2}$ and $f_{3}$ were used to resize the beam diameter to $3 \mathrm{~mm}$. Two $4 f$ systems were used to place the $\mathrm{SLM}_{2}, \mathrm{DM}$ and 


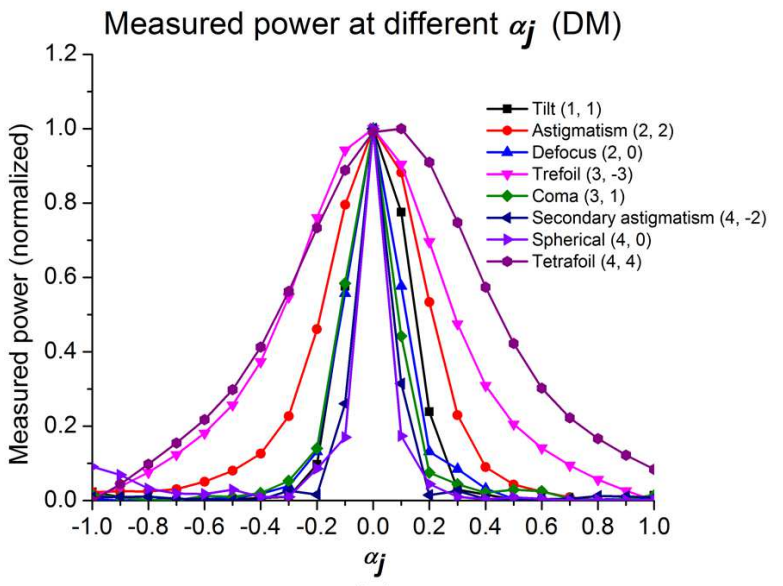

(a)

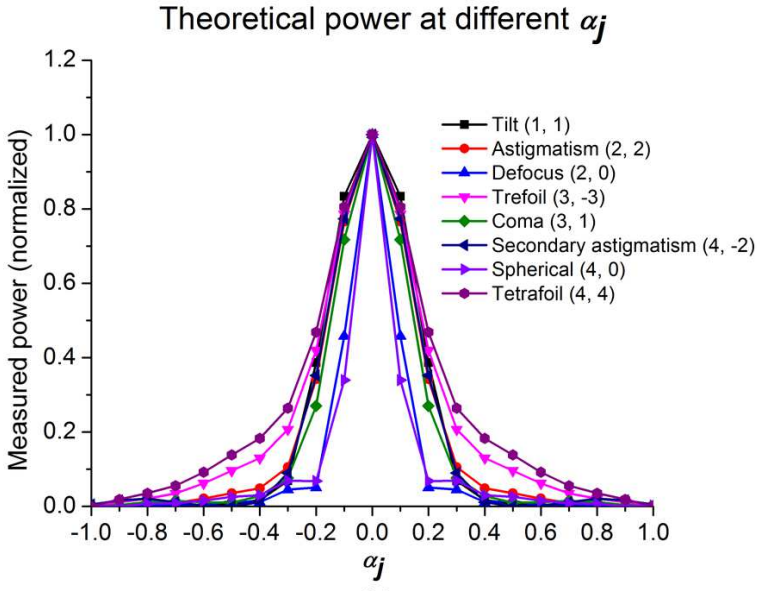

(b)

FIG. 3 (a) Experimentally measured power against $\alpha_{j}$ for the DM case. (b) Theoretical power as a function of $\alpha_{j} . \alpha$ represents the magnitude of the aberrations generated by the DM.

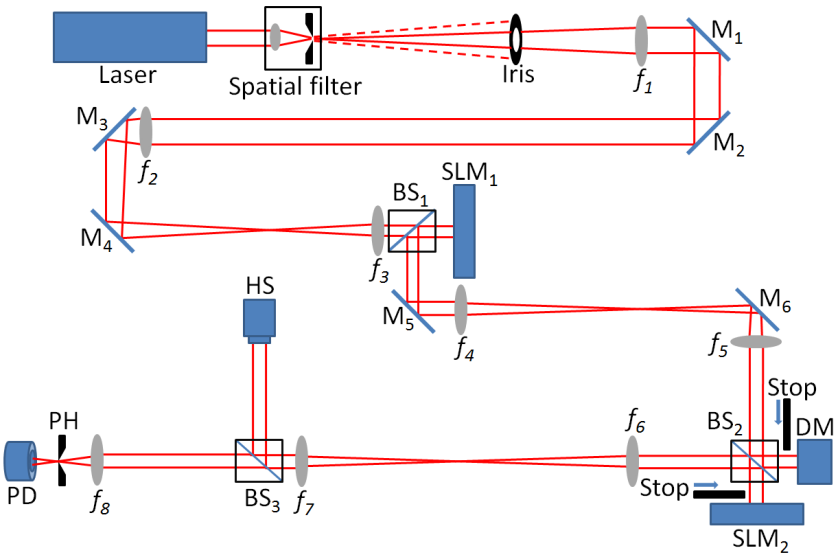

FIG. 2 Experimental setup to compare the performance of a DM and an SLM in signalbased wavefront sensing. All lenses are AR-coated achromats.

HS WFS in conjugate planes of $\mathrm{SLM}_{1}$. For wavefront sensing, the light was focused by a lens $\left(f_{8}=60 \mathrm{~mm}\right)$ on to a $25 \mu \mathrm{m}$ pinhole $(\mathrm{PH})$ which is $19 \%$ smaller than the Airy disc diameter. This choice of the pinhole size allows collecting enough light and being sensitive to aberrations. A photodetector (PD, PM 100D, S120C, Thorlabs, USA) placed behind the pinhole was used to detect the wavefront related signal.

An in-house developed LabVIEW program was run with three loops for the correction. The results of both correctors are discussed in Section 3 and Section 4 respectively. The RMS wavefront error values were calculated after correction by using, $\mathrm{RMS}=\sqrt{\sum_{i=1}^{i=14} a_{i}^{2}}$ and are shown in the figure captions. The RMS values reduce $\sim 18 \%$, if tilt coefficients are removed in the RMS calculation.

\section{DM-BASED AO SYSTEM}

Modal wavefront correction allows to control all the DM actuators simultaneously and hence is a faster alternative to wavefront correction than using individual actuators or zonal correction. During the correction, a maximum admissible voltage matrix was introduced that provides maximum DM deflection for a particular Zernike mode. Then the deflection was reduced in small steps to identify the best configuration that gives the highest detected power at the photodetector.

For the experimental verification of the capability of the DM in correcting various modes, the normalized measured powers as a function of the search parameter, $\alpha_{j}$ for individual Zernike modes were plotted. A flat wavefront was introduced by $\mathrm{SLM}_{1}$ after removing its own aberrations and different Zernike modes were introduced with the DM by controlling the actuator voltages, written in the form of an array, $\mathbf{V}_{a p p}$ and given the following equation:

$$
\mathbf{V}_{a p p}=\boldsymbol{\alpha}_{j} \cdot\left[\mathbf{V}_{\max }-\mathbf{V}_{0}\right]+\mathbf{V}_{0}
$$

where $\mathbf{V}_{\text {max }}$ is the maximum voltage array applied onto the actuators for a particular mode, and $\mathbf{V}_{0}$ is a voltage array corresponding to $50 \%$ bias stroke of the DM. The parameter $\alpha_{j}$ sets the magnitude of the applied aberration. Finally, the power was measured as a function of $\alpha_{j}, \forall-1 \leq \alpha_{j} \leq 1$. Here, $\alpha=1$ corresponds to peak-to-valley $(\mathrm{P}-\mathrm{V})$ of $3.3 \mu \mathrm{m}$.

Figure 3(a) shows plots of experimentally measured powers for different modes. It can be noted that maximum power is measured near $\boldsymbol{\alpha}_{j}=0$. For comparison, simulations were also performed to obtain theoretical plots as a function of normalized measured power as shown in Figure 3(b). The limited degrees of freedom and influence function of the DM, make it difficult to generate some of the Zernike modes. Therefore, the curves of measured power as function of $\boldsymbol{\alpha}_{j}$ for astigmatism $\left(Z_{2}^{2}\right)$, trefoil $\left(Z_{3}^{-3}\right)$ and tetrafoil $\left(Z_{4}^{4}\right)$ are broader in comparison with their theoretical counterparts.

The correction process was run by adopting a certain order. Defocus $\left(Z_{2}^{0}\right)$ was removed as a first step followed by tilt $\left(Z_{1}^{-1}\right.$ and $\left.Z_{1}^{1}\right)$, astigmatism $\left(Z_{2}^{-2}\right.$ and $\left.Z_{2}^{2}\right)$, coma $\left(Z_{3}^{-1}\right.$ and $\left.Z_{3}^{1}\right)$, trefoil $\left(Z_{3}^{-3}\right.$ and $\left.Z_{3}^{3}\right)$, spherical $\left(Z_{4}^{0}\right)$, tetrafoil $\left(Z_{4}^{-4}\right)$, secondary astigmatism $\left(Z_{4}^{-2}\right.$ and $\left.Z_{4}^{2}\right)$, and tetrafoil $\left(Z_{4}^{4}\right)$.

\subsection{Results}

Defocus $\left(Z_{2}^{0}\right)$, astigmatism $\left(Z_{2}^{2}\right)$, trefoil $\left(Z_{3}^{-3}\right)$ and randomlygenerated aberrations were tested in the DM-based wavefront correction system. Three different P-V: $1.00 \mu \mathrm{m}, 1.50 \mu \mathrm{m}$ 
Before correction of Defocus $(2,0)$

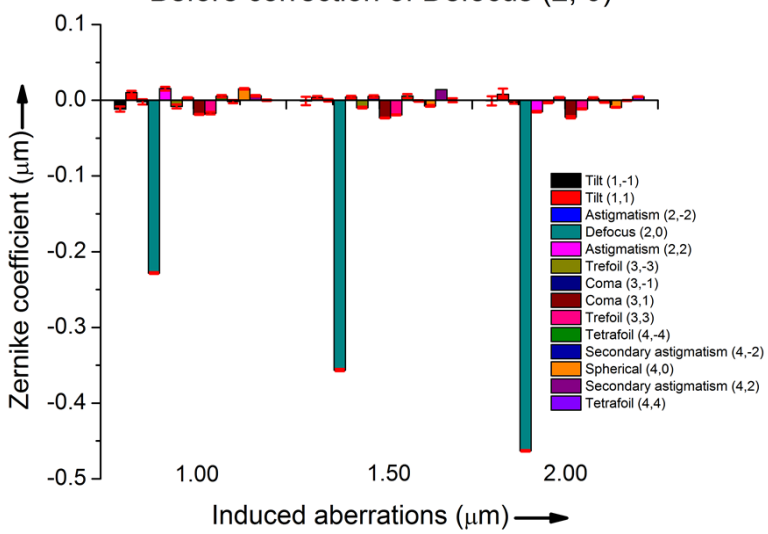

(a)

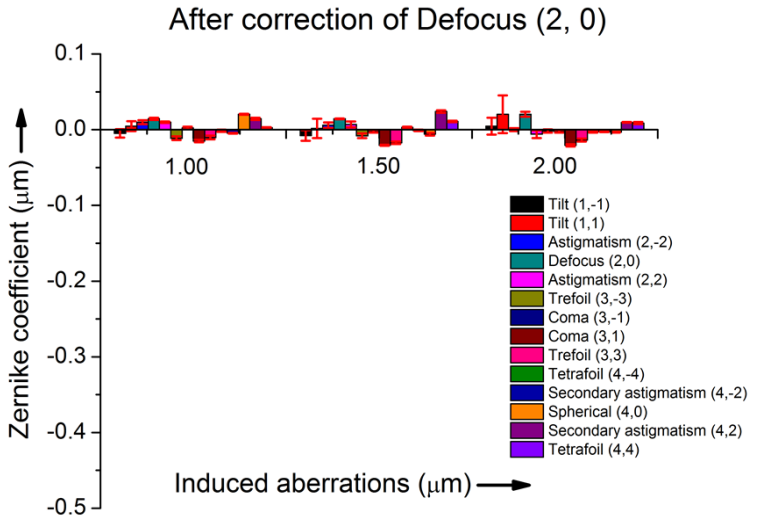

(b)

FIG. 4 HS measured Zernike coefficients for the DM case: (a) SLM 1 generated defocus of P-V : $1.00 \mu \mathrm{m}, 1.50 \mu \mathrm{m}$ and $2.00 \mu \mathrm{m}$ (b) Defocus including other aberrations have been removed after correction by the DM. The calculated RMS error equals $0.04 \mu \mathrm{m}$ after correction for all three cases shown. Error-bars show the standard deviation of a total of three measurement series.

Before correction of Astigmatism (2, 2)

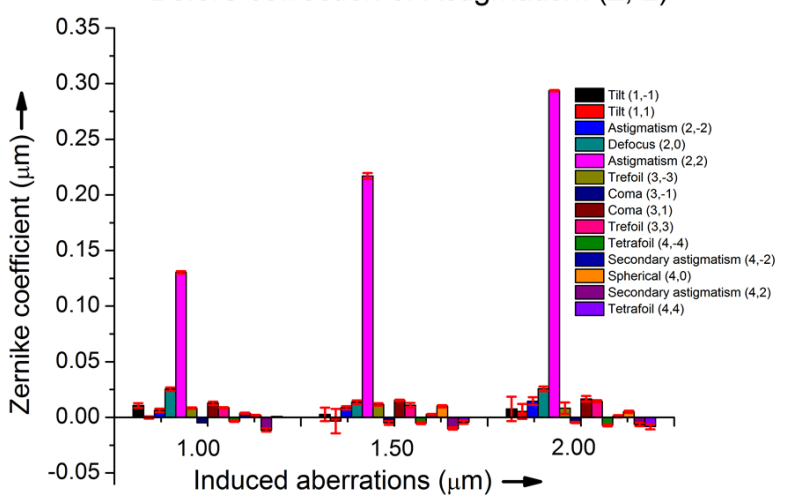

(a)

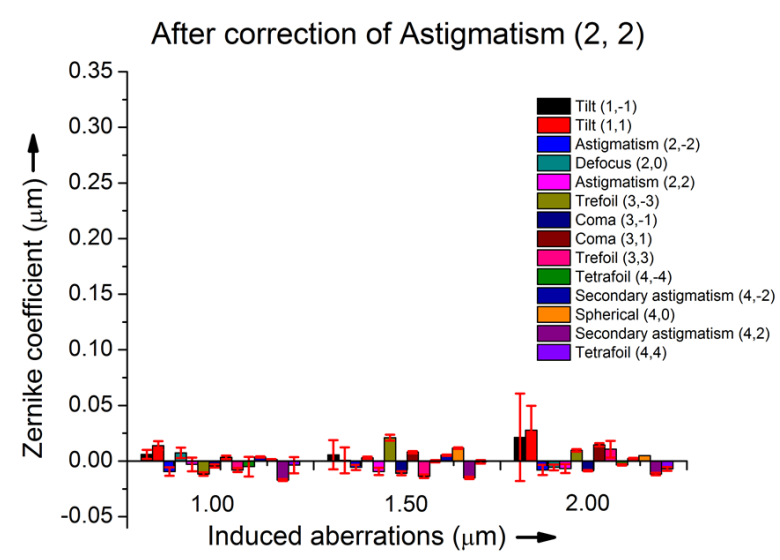

(b)

FIG. 5 HS measured Zernike coefficients for the DM case: (a) SLM 1 generated astigmatism ( $Z_{2}^{2}$ ) of P-V : $1.00 \mu \mathrm{m}, 1.50 \mu \mathrm{m}$ and $2.00 \mu \mathrm{m}$ (b) 0ther aberrations including dominant astigmatism $\left(Z_{2}^{2}\right)$ have been removed after correction by the DM. The calculated RMS errors are $0.03 \mu \mathrm{m}(\mathrm{P}-\mathrm{V} 1.00 \mu \mathrm{m}), 0.04 \mu \mathrm{m}(\mathrm{P}-\mathrm{V} 1.50 \mu \mathrm{m})$ and $0.05 \mu \mathrm{m}(\mathrm{P}-\mathrm{V} 2.00 \mu \mathrm{m})$ after correction.

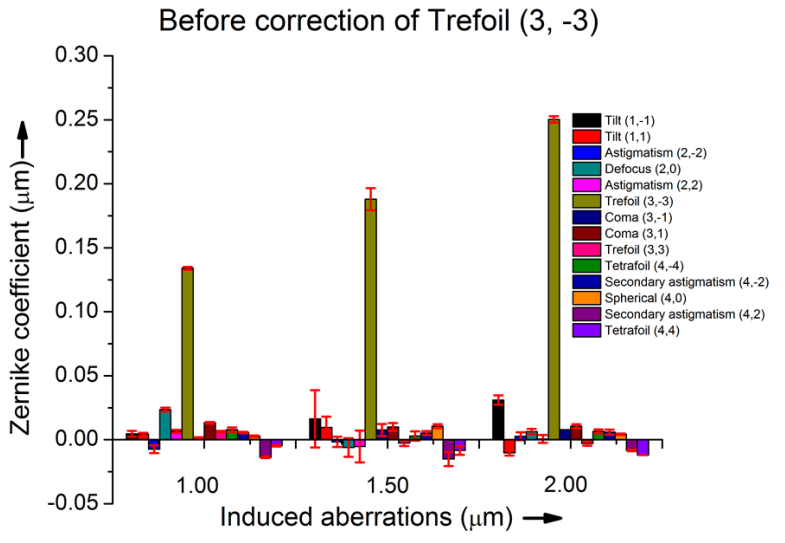

(a)

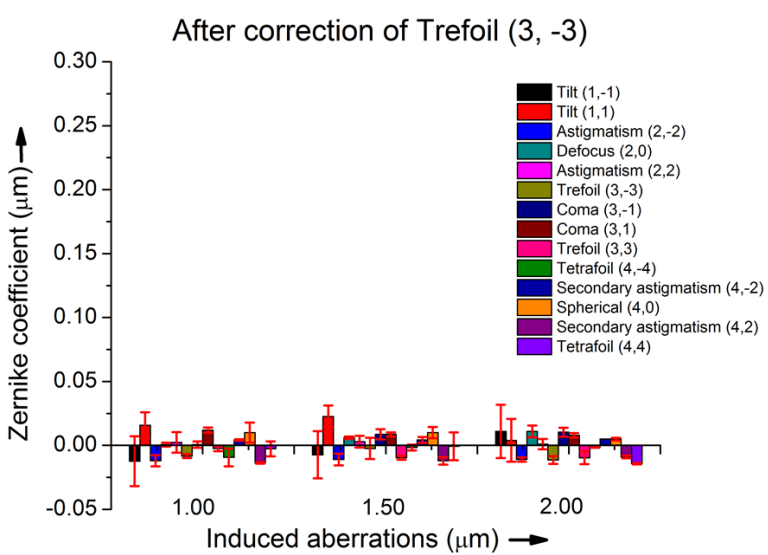

(b)

FIG. 6 HS measured Zernike coefficients for the DM case: (a) Trefoil $\left(Z_{3}^{-3}\right)$ of P-V : $1.00 \mu \mathrm{m}, 1.50 \mu \mathrm{m}$ and $2.00 \mu \mathrm{m}$ were induced by SLM ${ }_{1}$ (b) Trefoil $\left(Z_{3}^{-3}\right)$ including other aberrations have been removed after correction by the DM. The calculated RMS error equals $0.04 \mu \mathrm{m}$ after correction for all three cases shown.

and $2.00 \mu \mathrm{m}$ of each aberration were introduced in the system by using $\mathrm{SLM}_{1}$. The wavefront aberrations in terms of the Zernike coefficients measured by the HS are plotted in Figure 4 before and after the correction of defocus using three loops of power optimization.
HS measured Zernike coefficients before correction of astigmatism $\left(Z_{2}^{2}\right)$, trefoil $\left(Z_{3}^{-3}\right)$ and randomly-generated aberrations, introduced by $\mathrm{SLM}_{1}$ are shown in Figure 5(a), 6(a) and 7(a) respectively. Zernike coefficients were again measured after running three loops of wavefront correction and are shown in Figure 5(b), 6(b) and 7(b) respectively. The time taken to 


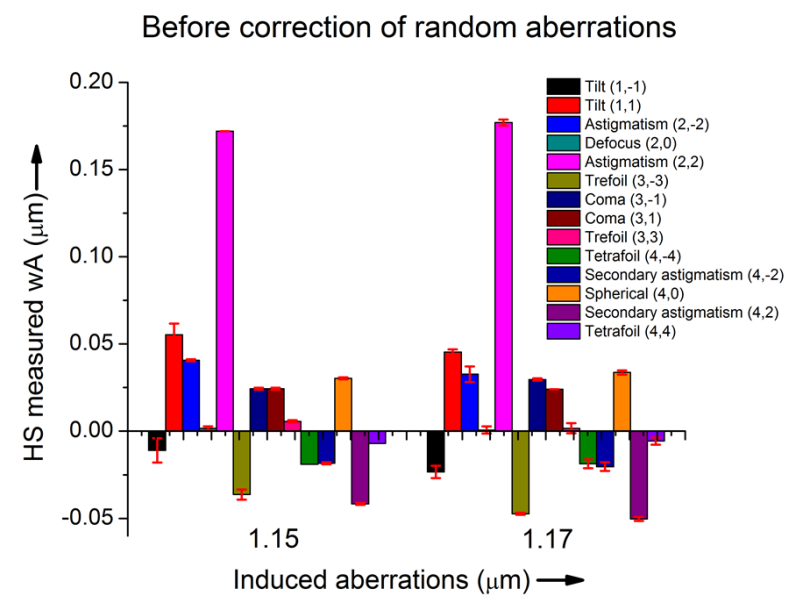

(a)

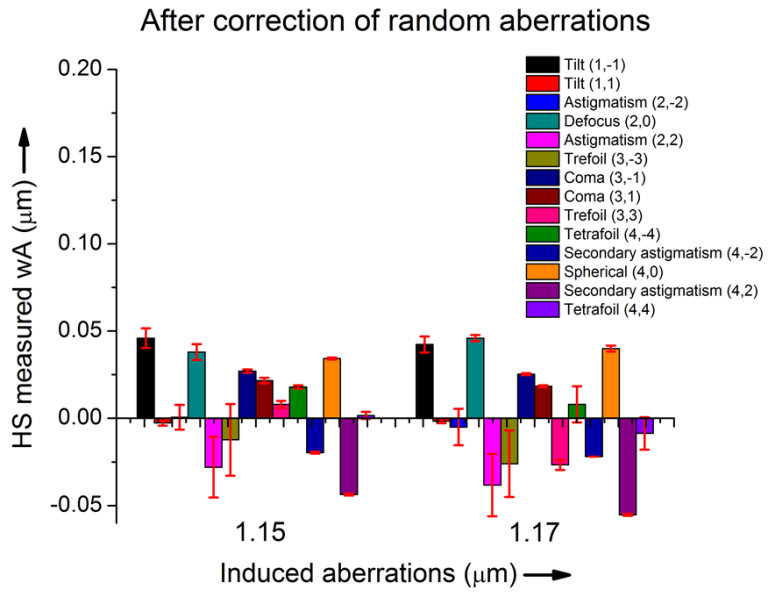

(b)

FIG. 7 HS measured Zernike coefficients for the DM case: (a) SLM 1 generated random aberrations of P-V : $1.15 \mu \mathrm{m}$ and $1.17 \mu \mathrm{m}$ (b) After correction by the DM. The calculated RMS errors are $0.10 \mu \mathrm{m}\left(\mathrm{P}_{-} \mathrm{V} 1.15 \mu \mathrm{m}\right)$ and $0.12 \mu \mathrm{m}$ ( $\mathrm{P}-\mathrm{V}_{1.17 \mu \mathrm{m})}$ after correction.

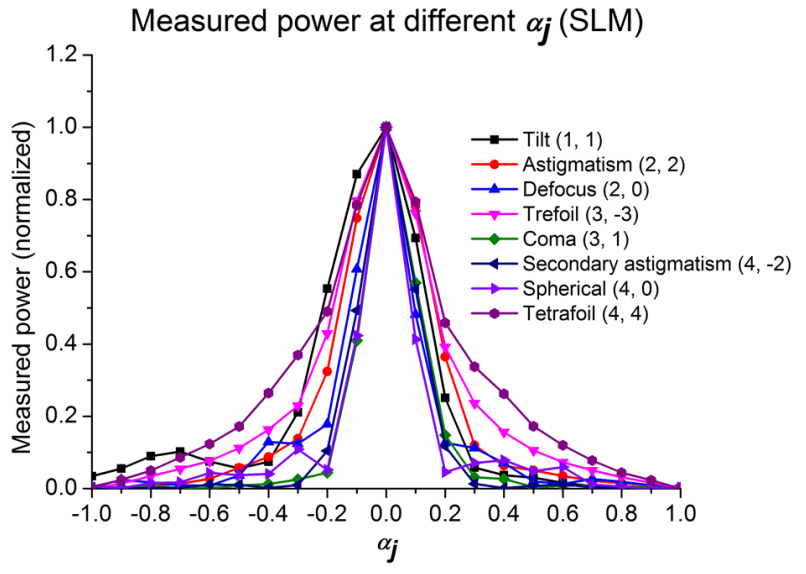

(a)

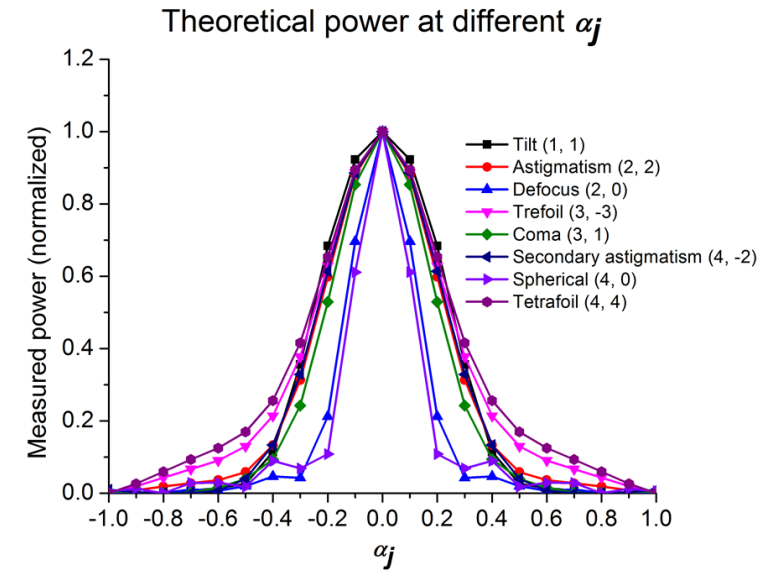

(b)

FIG. 8 The graph of measured power against $\alpha_{j}$. (a) Experimentally measured power against $\alpha_{j}$ for the SLM case. (b) Theoretical power as a function of $\alpha_{j}$. $\alpha$ represents the magnitude of the aberrations generated by the $\mathrm{SLM}_{2}$.

complete each wavefront correction is approximately seven minutes due to the three loops and the large number of $\boldsymbol{\alpha}_{j}$ steps. All measurements were repeated three times to determine the mean and standard deviation.

\section{SLM-BASED AO SYSTEM}

Power measurements similar to those taken with the DM were performed with the $\mathrm{SLM}_{2}$-based AO system. After introducing a uniform phase matrix of zero gray values on both SLMs, small amount of astigmatism and trefoil were measured by the HS. Therefore, a correction matrix $\left(\mathbf{Z}_{c}\right)$, calculated from the signal based WFS with a small step size, was added to $\mathrm{SLM}_{2}$ to compensate these static aberrations. The power increased by nearly $50 \%$ after the introduction of the correction matrix $\left(\mathbf{Z}_{c}\right)$ confirming that the SLM is also a source of aberrations if not corrected precisely. To obtain the graph of measured power as a function of $\boldsymbol{\alpha}_{j}$ for the SLM case, a flat wavefront was introduced by $\mathrm{SLM}_{1}$ and individual Zernike modes of different magnitude were introduced on $\mathrm{SLM}_{2}$. The applied phase matrix for different aberration modes were calculated by using the following equation:

$$
\mathbf{Z}_{a p p}=\boldsymbol{\alpha}_{j} \cdot \mathbf{Z}_{\max }+\mathbf{Z}_{\mathcal{C}}
$$

where $\mathbf{Z}_{a p p}$ is the matrix of applied phase values, $\mathbf{Z}_{\max }$ is the phase matrix with the maximum allowed values for a particular mode and $\mathbf{Z}_{c}$ is the SLM correction matrix. The parameter $\boldsymbol{\alpha}_{j}, \forall-1 \leq \boldsymbol{\alpha}_{j} \leq 1$ is used to alter the magnitude of $\mathbf{Z}_{a p p}$.

To avoid an overlap with the unmodulated zeroth diffraction order, a blazed grating with a small period of $60 \mu \mathrm{m}$ (equal to 3 pixels) was used and the measurements were performed using the first diffraction order. The measured first order diffraction efficiency was 0.26 .

Figure 8 shows the change of experimentally measured signal with different $\alpha_{j}$ where $\alpha_{j}=1$ corresponds to P-V of $2.27 \mu \mathrm{m}$. The plots are in good agreement with the theoretical predictions and a maximum power was achieved at $\boldsymbol{\alpha}_{j}=0$ which confirms the absence of any aberrations in significant quantities and accuracy of determining the correction matrix.

\subsection{Results}

Similar to the DM wavefront correction system, $\mathrm{SLM}_{1}$ was employed to induce aberrations and $\mathrm{SLM}_{2}$ was used for correction. The Zernike coefficients before and after wavefront correction were measured by the HS. Since the response time of 
Before correction of Defocus $(2,0)$

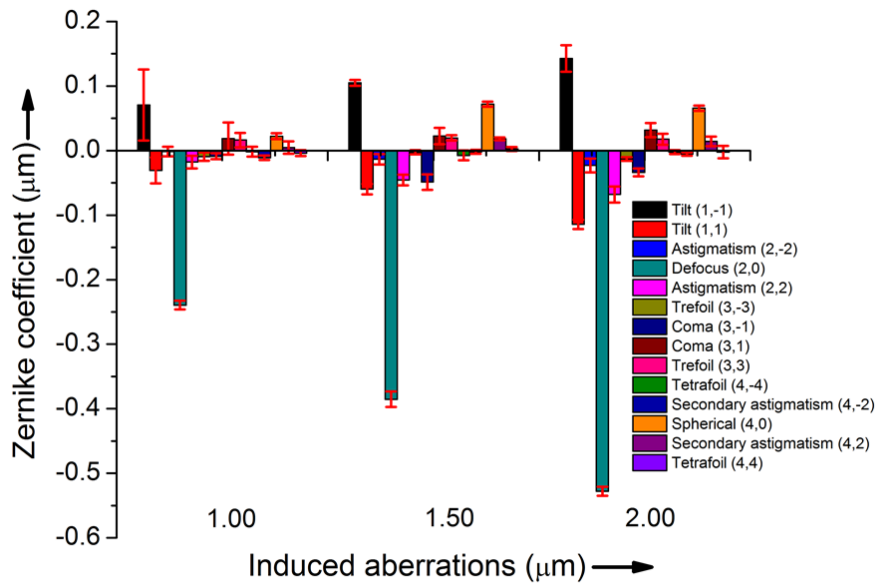

(a)

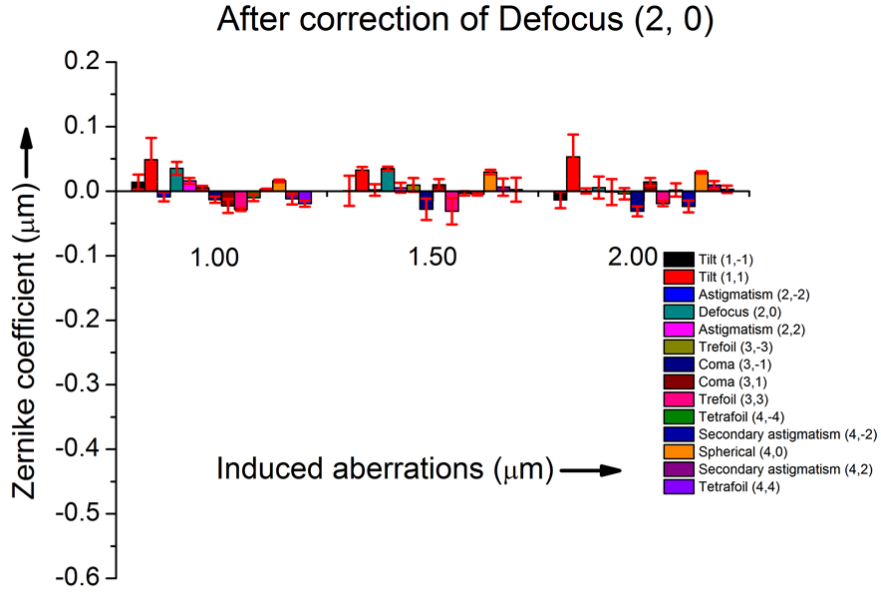

(b)

FIG. 9 HS measured Zernike coefficients for the SLM case: (a) SLM 1 generated defocus of P-V : $1.00 \mu \mathrm{m}, 1.50 \mu \mathrm{m}$ and $2.00 \mu \mathrm{m}$ (b) Defocus including other aberrations have been removed by $\mathrm{SLM}_{2}$ after correction. The calculated RMS errors are $0.09 \mu \mathrm{m}$ (P-V $\left.1.00 \mu \mathrm{m}\right), 0.08 \mu \mathrm{m}(\mathrm{P}-\mathrm{V} 1.50 \mu \mathrm{m})$ and $0.09 \mu \mathrm{m}$ (P-V $\left.2.00 \mu \mathrm{m}\right)$ after correction.

Before correction of Astigmatism (2, 2)

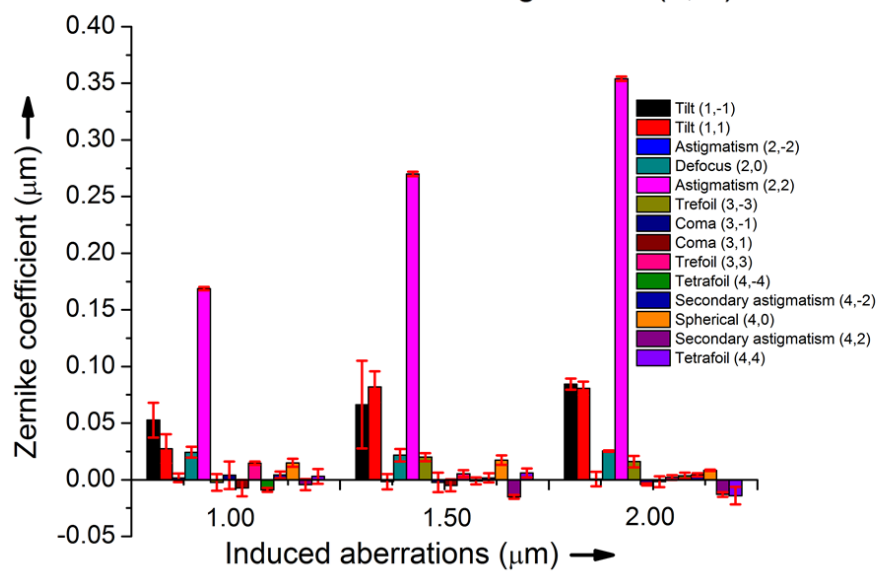

(a)

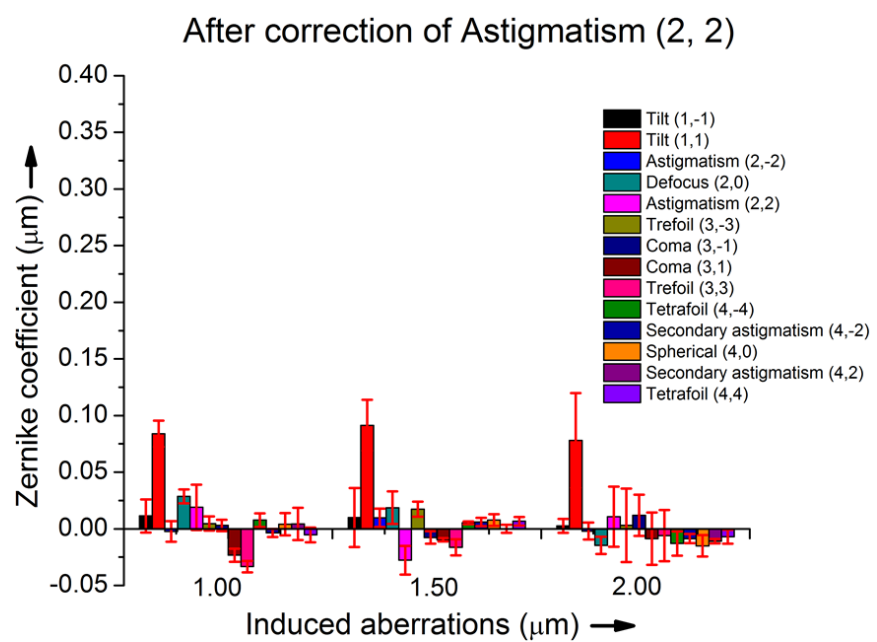

(b)

FIG. $10 \mathrm{HS}$ measured Zernike coefficients for the SLM case: (a) SLM 1 generated astigmatism $\left(Z_{2}^{2}\right)$ of P-V : $1.00 \mu \mathrm{m}, 1.50 \mu \mathrm{m}$ and $2.00 \mu \mathrm{m}$ (b) 0ther aberrations including dominant astigmatism $\left(Z_{2}^{2}\right)$ have been removed after correction by SLM . The calculated RMS errors are $0.10 \mu \mathrm{m}(\mathrm{P}-\mathrm{V} 1.00 \mu \mathrm{m}), 0.10 \mu \mathrm{m}(\mathrm{P}-\mathrm{V} 1.50 \mu \mathrm{m})$ and $0.09 \mu \mathrm{m}(\mathrm{P}-\mathrm{V} 2.00 \mu \mathrm{m})$ after running the correction.

the SLM is $\sim 60 \mathrm{~Hz}$ as compared to the $\sim 2 \mathrm{kHz}$ of the DM, it requires approximately fifteen minutes to complete the full correction process for the chosen number of twenty-one steps and three loops.

Figure 9(a) shows the dominance of defocus before the wavefront correction when $\mathrm{SLM}_{1}$ induced defocus of P-V $1.00 \mu \mathrm{m}$, $1.50 \mu \mathrm{m}$ and $2.00 \mu \mathrm{m}$ in the system consecutively. Figure $9(\mathrm{~b})$ shows the Zernike coefficient values measured after running the wavefront correction process.

The plot in Figure 10(a) shows the Zernike coefficient values while astigmatism $\left(Z_{2}^{2}\right)$ was induced in the system for three different P-V of $1.00 \mu \mathrm{m}, 1.50 \mu \mathrm{m}$ and $2.00 \mu \mathrm{m}$ before correction. Though astigmatism $\left(Z_{2}^{2}\right)$ alone was induced, a small magnitude of other aberrations i.e. tilt $\left(Z_{1}^{-1}\right)$ and $\left(Z_{1}^{1}\right)$ were noted. After running the wavefront correction, Zernike coefficient values measured by the HS were plotted in Figure 10(b). Most of the dominant aberrations were removed after correc- tion except tilt $\left(Z_{1}^{1}\right)$. This is likely caused by small mechanical shifts of the HS mounting base during the prolonged measurements.

Figure 11(a) shows the Zernike coefficient values after introducing trefoil $\left(Z_{3}^{-3}\right)$ in the system by $\mathrm{SLM}_{1}$ for three different P-V of $1.00 \mu \mathrm{m}, 1.50 \mu \mathrm{m}$ and $2.00 \mu \mathrm{m}$. Figure 11(b) shows the Zernike coefficient values after correcting dominant trefoil $\left(Z_{3}^{-3}\right)$ including other aberration by $\mathrm{SLM}_{2}$.

We checked the correction process for randomly-generated aberrations. Figure 12(a) shows two randomly generated aberrations by $\mathrm{SLM}_{1}$ of $\mathrm{P}-\mathrm{V}: 1.15 \mu \mathrm{m}$ and $1.17 \mu \mathrm{m}$. Figure 12(b) shows the Zernike coefficients values after the correction.

\section{DISCUSSION AND CONCLUSION}

The measured Zernike coefficients before and after correction were employed to calculate the on-axis Strehl ratio. The same 


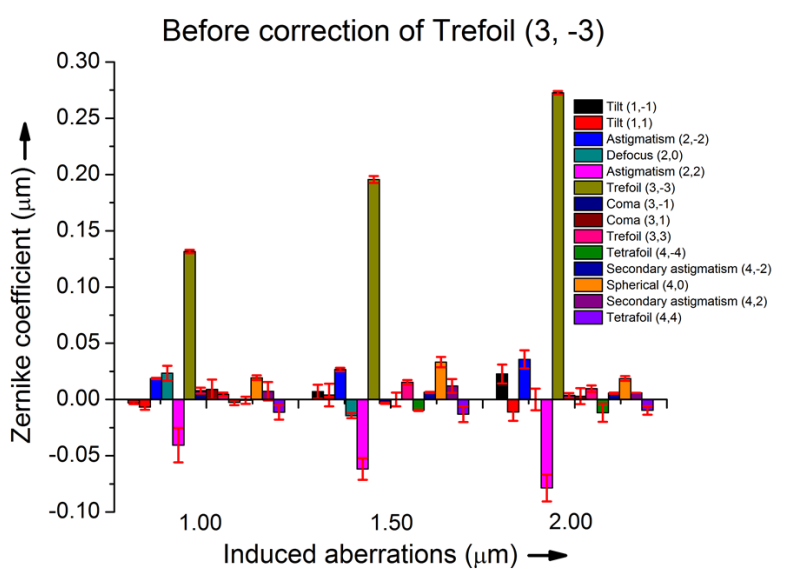

(a)

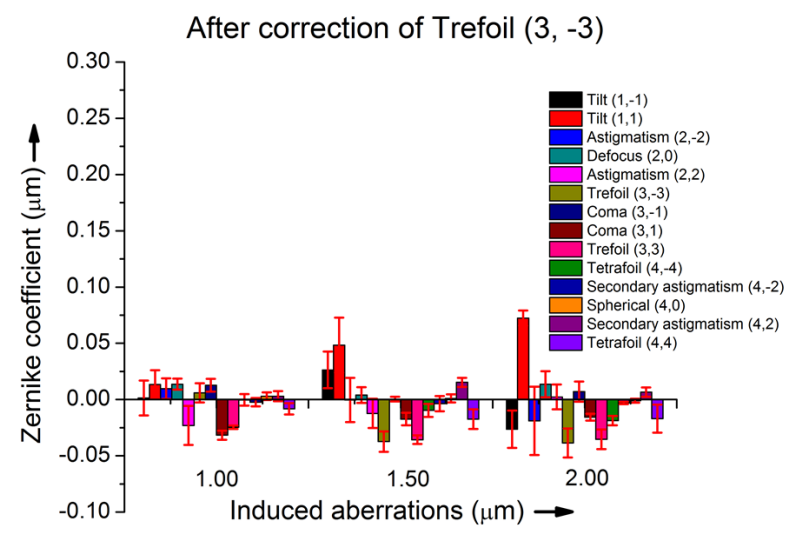

(b)

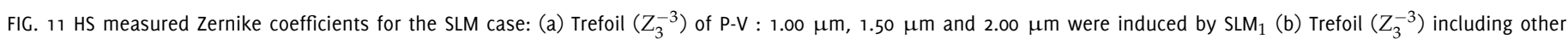
aberrations have been removed by $\mathrm{SLM}_{2}$ based on the detected power. The calculated RMS errors are $0.06 \mu \mathrm{m}(\mathrm{P}-\mathrm{V} 1.00 \mu \mathrm{m}), 0.08 \mu \mathrm{m}(\mathrm{P}-\mathrm{V} 1.50 \mu \mathrm{m})$ and $0.1 \mu \mathrm{m}(\mathrm{P}-\mathrm{V} 2.00 \mu \mathrm{m})$ after correction.

Before correction of random aberrations

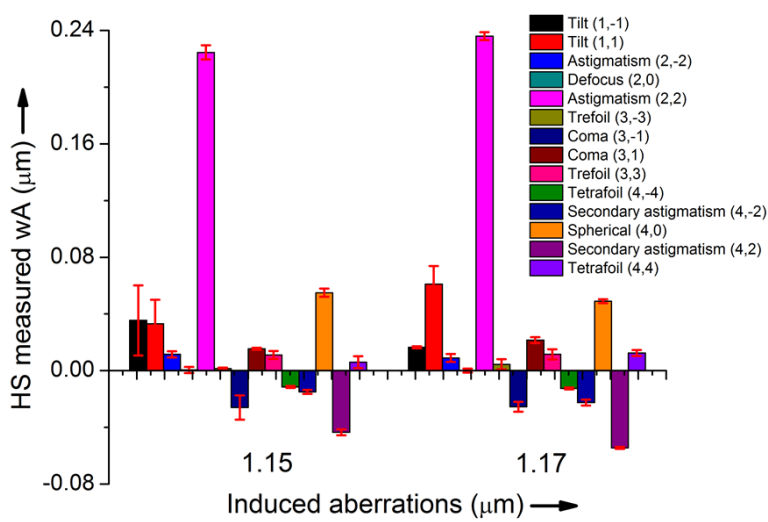

(a)
After correction of random aberrations

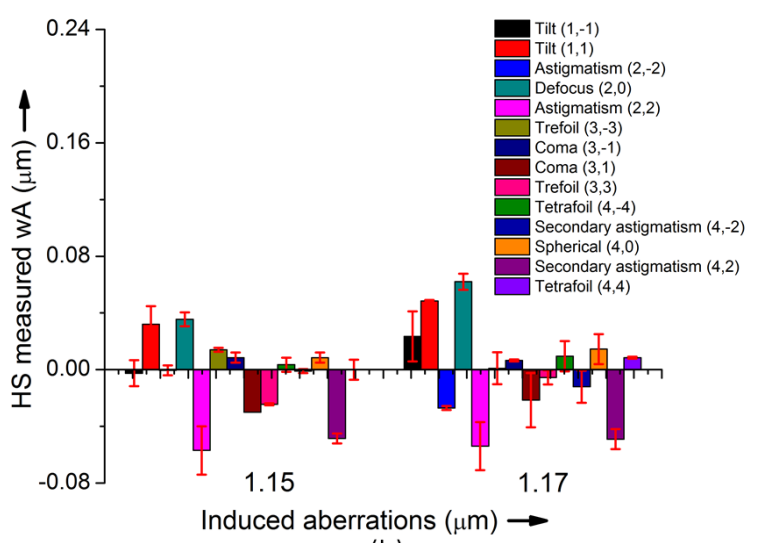

(b)

FIG. 12 HS measured Zernike coefficients for the SLM case: (a) SLM 1 generated random aberrations of P-V : $1.15 \mu \mathrm{m}$ and $1.17 \mu \mathrm{m}$ (b) After correction by SLM 2 based on the detected power. The calculated RMS errors after correcting the aberrations are $0.10 \mu \mathrm{m}$ ( $\mathrm{P}-\mathrm{V} 1.15 \mu \mathrm{m})$ and $0.12 \mu \mathrm{m}(\mathrm{P}-\mathrm{V} 1.17 \mu \mathrm{m})$.

magnitude of aberrations were induced in both systems to compare the performance of the DM and the SLM. Both devices show a good improvement in Strehl ratios after the correction of the induced aberrations (Figures 13-16). The tilt coefficients were not included in calculating the Strehl ratios as tilt terms do not effect the point spread function.

Although the SLM was operated in phase-only mode, there could be minor amplitude modulation errors. A blazed grating was used to avoid unmodulated zeroth diffraction order and a correction matrix was used to remove its own pattern of aberrations.

Implementation of a Gaussian interpolation in combination with normal search has enhanced the correction ability and speed. It was observed that the DM has used more Gaussian interpolated results compared to the SLM. Quadratic interpolation was also implemented and the results were similar to that obtained with Gaussian interpolation.

The improvement in Strehl ratio for the case of defocus is shown in Figure 13. With the DM, calculated Strehl ratios were $3.02 \times 10^{-2}, 1.60 \times 10^{-3}$ and $1.36 \times 10^{-2}$ before the correction and $0.87,0.84,0.88$ after the correction of dominant defocus of
$\mathrm{P}-\mathrm{V}: 1.00 \mu \mathrm{m}, 1.50 \mu \mathrm{m}$ and $2.00 \mu \mathrm{m}$ respectively. The Strehl ratios in case of the SLM for similar P-V as in the DM case were $0.03,7.70 \times 10^{-3}$ and $1.90 \times 10^{-3}$ before correction and $0.66,0.63,0.69$ after correction respectively. The improvement in Strehl ratio for astigmatism $\left(Z_{2}^{2}\right)$ is shown in Figure 14. In the DM case, the Strehl ratios for the P-V of $1.00 \mu \mathrm{m}, 1.50 \mu \mathrm{m}$ and $2.00 \mu \mathrm{m}$ were $0.16,7.50 \times 10^{-3}$ and $1.21 \times 10^{-2}$ before correction and $0.92,0.88$ and 0.91 after correction respectively. For the SLM, the Strehl ratios were $0.06,4.60 \times 10^{-3}$ and 0.02 before correction and $0.72,0.79$ and 0.72 after correction respectively. As it is difficult to introduce a pure Zernike mode at a time as sensed by the HS WFS, other minor Zernike coefficients will tend to be present. Therefore, the calculated Strehl ratios before correction for P-V: $2.0 \mu \mathrm{m}$ were higher than for $\mathrm{P}-\mathrm{V}: 1.5 \mu \mathrm{m}$ as seen in Figure 13(a) and Figure 14.

The improvement in Strehl ratio for trefoil is shown in Figure 15. The Strehl ratio for P-V of $1.00 \mu \mathrm{m}, 1.50 \mu \mathrm{m}$ and $2.00 \mu \mathrm{m}$ for trefoil $\left(Z_{3}^{-3}\right)$ were $0.20,0.09,0.07$ before correction and $0.91,0.92,0.90$ after correction respectively for the DM case. For similar amount of trefoil $\left(Z_{3}^{-3}\right)$ in case of the SLM, the Strehl ratios were $0.19,0.07,0.07$ before correction and $0.74,0.66,0.63$ after correction. 
Improvement of Strehl ratio (DM)

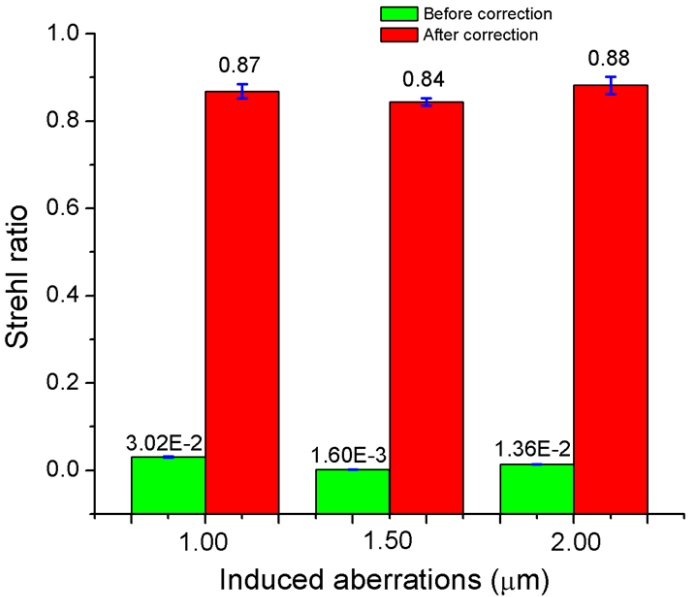

(a)
Improvement of Strehl ratio (SLM)

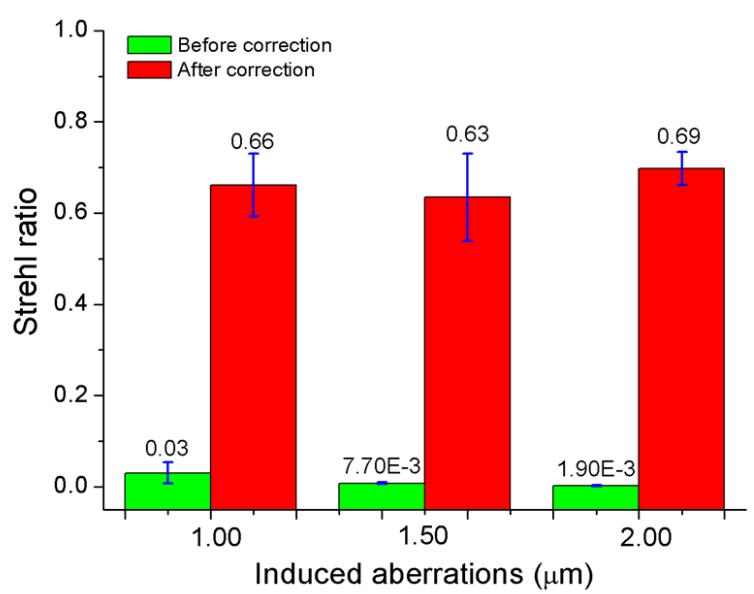

(b)

FIG. 13 Improvement of Strehl ratio before and after correction of defocus $\left(Z_{2}^{0}\right)$ is shown for (a) the DM and (b) the SLM system. In both plots, two colors Green and Red are indicating the Strehl ratios before and after the correction respectively.

Improvement of Strehl ratio (DM)

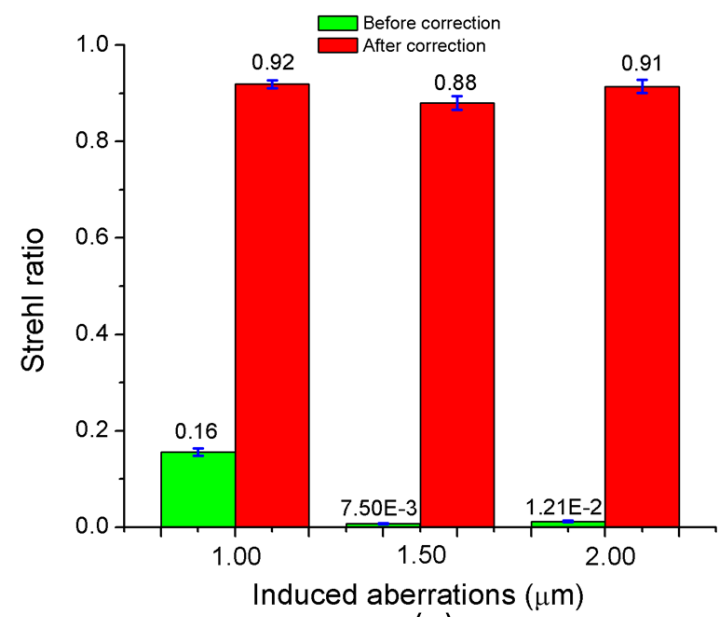

(a)

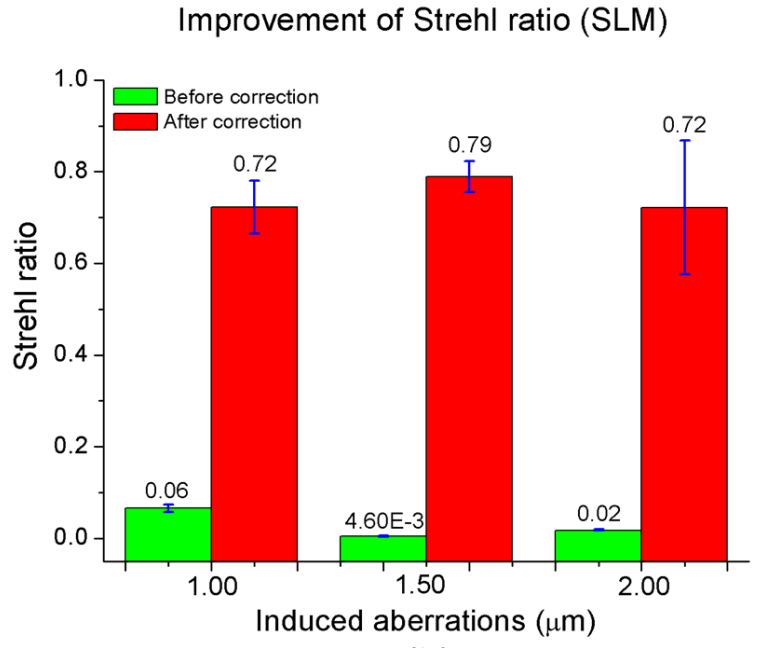

(b)

FIG. 14 Improvement of Strehl ratio before and after correction of astigmatism $\left(Z_{2}^{2}\right)$ is shown for (a) the DM and (b) the SLM system. Green and Red are indicating the Strehl ratios before and after the correction respectively.

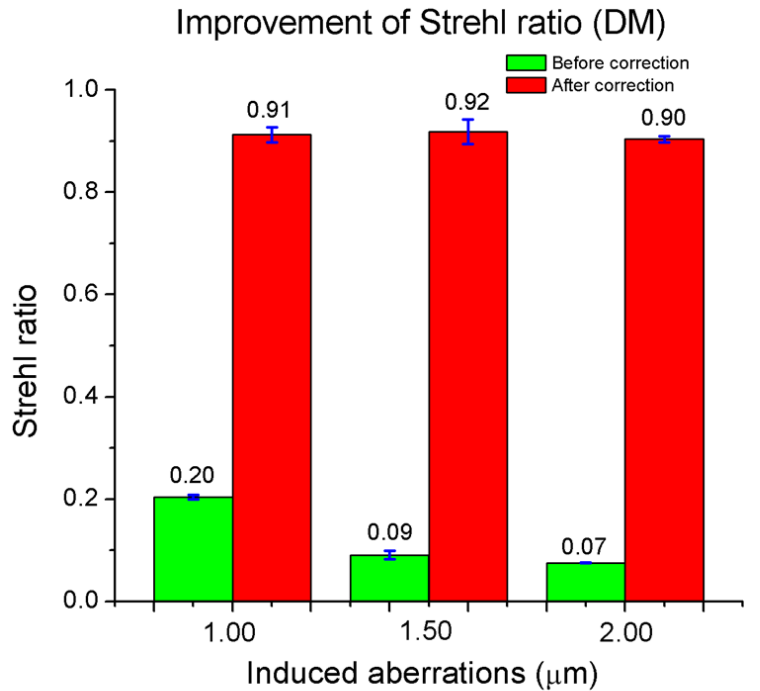

(a)

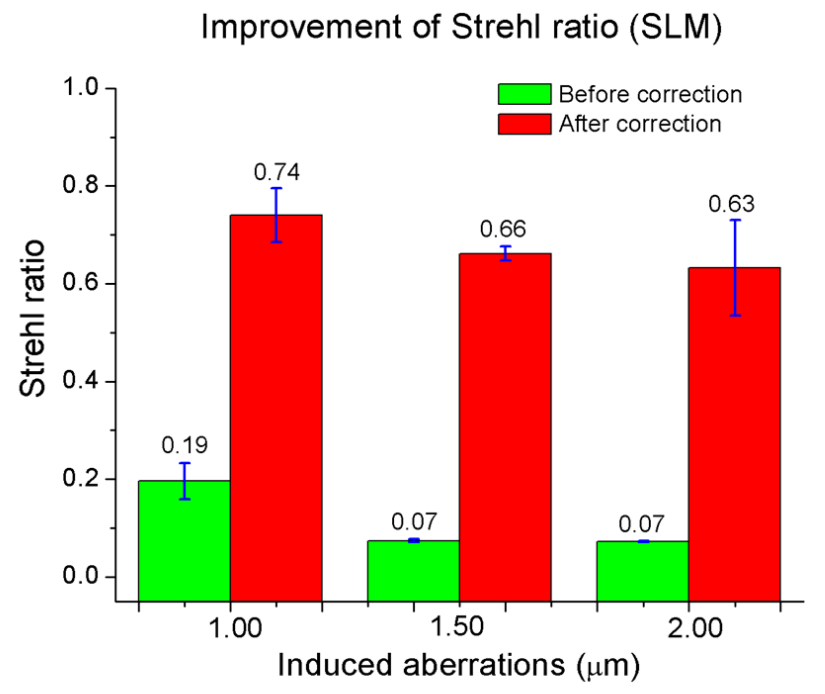

(b)

FIG. 15 Improvement of Strehl ratio before and after correction of trefoil $\left(Z_{3}^{-3}\right)$ is shown for (a) the DM and (b) the SLM system. Green and Red are indicating the Strehl ratios before and after the correction respectively. 


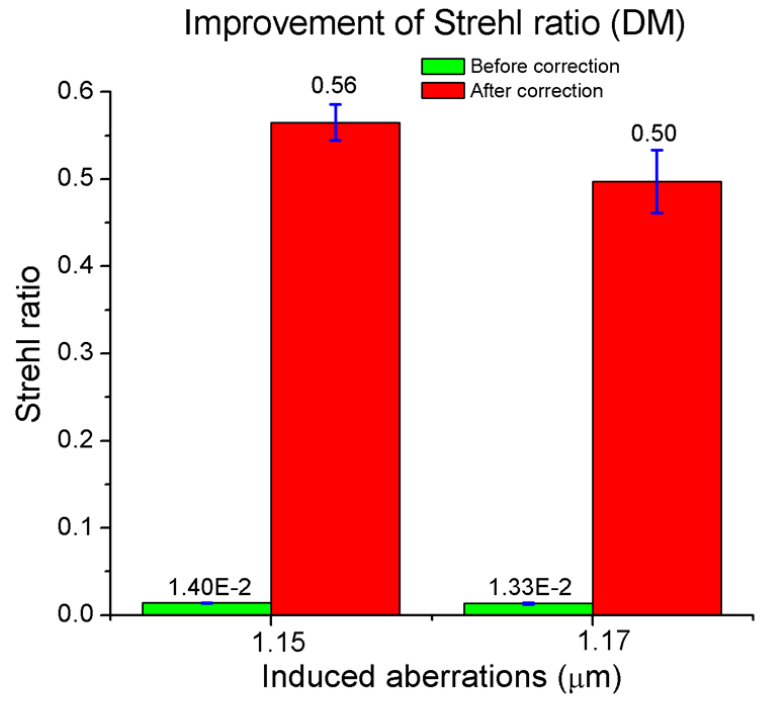

(a)

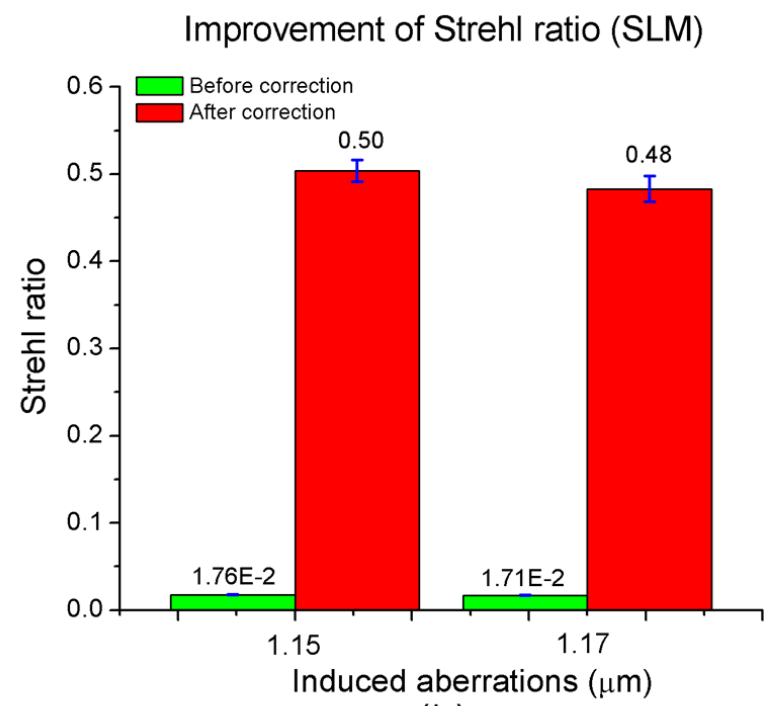

(b)

FIG. 16 Improvement of Strehl ratio before and after correction of randomly generated aberration is shown for (a) the DM and (b) the SLM system. Green and Red are indicating the Strehl ratios before and after the correction respectively.

The improvement in Strehl ratio for randomly generated aberrations is shown in Figure 16. The Strehl ratio for randomly generated aberrations of $\mathrm{P}-\mathrm{V}: 1.15 \mu \mathrm{m}$ and $1.17 \mu \mathrm{m}$ were $1.40 \times 10^{-2}$ and $1.33 \times 10^{-2}$ before correction for the DM case whereas $1.76 \times 10^{-2}$ and $1.71 \times 10^{-2}$ for the SLM case respectively. After correction the Strehl ratios were 0.56, 0.50 for the DM case and 0.50 and 0.48 for the SLM case respectively. The accuracy of wavefront correction can be improved further by increasing the number of loops and reducing the step size but at the cost of time.

In conclusion, the performance of two reflective wavefront correctors, a DM and an SLM have been compared for signalbased AO. It has been found that an SLM can be a promising alternative to the DM as the pixel resolution in SLM is higher than that of the DM. The DM used here can modulate the phase to a maximum of $22 \pi$ ( $3.5 \mu \mathrm{m}$ stroke) whereas the SLM can modulate the phase beyond $22 \pi$, which can be achieved by phase wrapping. However, phase modulation and wrapping by the SLM can generate losses through diffraction. At the chosen wavelength, the maximum phase amplitude of the SLM is $\sim 3 \pi$ when the phase is not wrapped. $150 \times 150$ pixels were used on the SLM to employ similar conditions as for the DM. With more pixels, the errors due to phase wrapping could be reduced while correcting higher order and large magnitude aberrations [23]. Zernike coefficients have also been measured after correction of astigmatism $\left(Z_{2}^{2}\right)$ by binning the SLM pixels such that $150 \times 150$ pixels acts as $12 \times 12$ pixels similar to the DM actuators. The Strehl ratio with binning was one order lower than without binning.

It was found that the DM can perform better than the SLM for the chosen number of pixels. The correction speed can potentially be accelerated using more advanced search algorithms for confocal and multiphoton microscopy where the use of HS WFS is problematic [24].

\section{ACKNOWLEDGEMENT}

The authors are thankful to Science Foundation Ireland (grants: 07/SK/B1239a and 08/In.1/B2053) for the financial support and Boston Micromachines for their help.

\section{A APPENDIX}

The Zernike polynomials $\left(Z_{n}^{m}\right)$ used in this paper are listed in Table 1 . The mode indexing schemes, using the single index $i$ or the dual indices $(n, m)$, are explained by J. Schwiegerling $[25,26] . r$ is the radial coordinate ranging from 0 to 1 and $\theta$ is the azimuthal component ranging from 0 to $2 \pi$.

\begin{tabular}{|l|l|l|l|l|}
\hline \multicolumn{3}{|c|}{ Index } & \multicolumn{2}{c|}{ Zernike mode } \\
\hline$i$ & $n$ & $m$ & $Z(r, \theta)$ & Aberration \\
\hline 1 & 1 & -1 & $2 r \sin (\theta)$ & Tilt \\
\hline 2 & 1 & 1 & $2 r \cos (\theta)$ & Tilt \\
\hline 3 & 2 & -2 & $2 \sqrt{3} r^{2} \sin (2 \theta)$ & Astigmatism \\
\hline 4 & 2 & 0 & $\sqrt{3}\left(2 r^{2}-1\right)$ & Defocus \\
\hline 5 & 2 & 2 & $2 \sqrt{3} r^{2} \cos (2 \theta)$ & Astigmatism \\
\hline 6 & 3 & -3 & $2 \sqrt{2} r^{3} \sin (3 \theta)$ & Trefoil \\
\hline 7 & 3 & -1 & $2 \sqrt{2}\left(3 r^{3}-2 r\right) \sin (\theta)$ & Coma \\
\hline 8 & 3 & 1 & $2 \sqrt{2}\left(3 r^{3}-2 r\right) \cos (\theta)$ & Coma \\
\hline 9 & 3 & 3 & $2 \sqrt{2} r^{3} \cos (3 \theta)$ & Trefoil \\
\hline 10 & 4 & -4 & $\sqrt{10} r^{4} \sin (4 \theta)$ & Tetrafoil \\
\hline 11 & 4 & -2 & $\sqrt{10} \quad\left(4 r^{4} \sin (2 \theta)-\right.$ & $\begin{array}{l}\text { Secondary } \\
\text { astigmatism }\end{array}$ \\
\hline 12 & 4 & 0 & $\sqrt{5} \sin (2 \theta))$ & Spherical \\
\hline 13 & 4 & 2 & $\begin{array}{l}\sqrt{10} \quad\left(4 r^{4} \cos (2 \theta)-\right. \\
\left.3 r^{2} \cos (2 \theta)\right)\end{array}$ & $\begin{array}{l}\text { Secondary } \\
\text { astigmatism }\end{array}$ \\
\hline 14 & 4 & 4 & $\sqrt{10} r^{4} \cos (4 \theta)$ & Tetrafoil \\
\hline
\end{tabular}

TABLE 1 Zernike polynomials. 


\section{References}

[1] F. S. Wouters, P. J. Verveer, and P. I. H. Bastaiens, “Imaging biochemistry inside cells," Trends Cell Biol. 11, 203-211 (2001).

[2] M. Peter, and S. M. Ameer-Beg, “Imaging molecular interactions by multiphoton FLIM," Biol. Cell 96, 231-236 (2004).

[3] M. J. Booth, D. Debarre, and A. Jesacher, "Adaptive Optics for Biomedical Microscopy," Opt. Photon. News 23, 22-29 (2012).

[4] X. Tao, B. Fernandez, 0. Azucena, M. Fu, D. C. Carcia, Y. Zuo, D. Chen, et al., "Adaptive optics confocal microscopy using direct wavefront sensing," Opt. Lett. 36, 1062-1064 (2011).

[5] J. B. Pawley, Handbook of biological confocal microscopy (Springer, New York, 2006).

[6] M. J. Booth, "Adaptive optics in microscopy," Philos. Trans. Soc. A $365,2829-2843$ (2007).

[7] J. W. Hardy, Adaptive Optics for Astronomical Telescopes (Oxford University Press, 0xford, 1998).

[8] M. Shaw, K. O'Holleran, and C. Paterson, "Investigation of the confocal wavefront sensor and its application to biological microscopy," Opt. Express 21, 19353-19362 (2013).

[9] G. Cao, and Xin Yu, "Accuracy analysis of a Hartmann-Shack wavefront sensor operated with a faint object," Opt. Eng. 33, 2331-2335 (1994).

[10] A. Vyas, M. B. Roopashree, and B. R. Prasad, “Advanced Methods for Improving the Efficiency of a Shack Hartmann Wavefront Sensor" in Topics in Adaptive Optics, R. K. Tyson, ed., 167-196 (InTech, Rijeka, 2012).

[11] M. J. Booth, "Wavefront sensor less adaptive optics for large aberrations," Opt. Express 32, 5-7 (2007).

[12] 0. Albert, L. Sherman, G. Mourou, T. B. Norris, and G. Vdovin, "Smart microscope: an adaptive optics learning system for aberration correction in multiphoton confocal microscopy," Opt. Lett. 25, 52-54 (2000).

[13] L. Sherman, J. Y. Ye, 0. Albert and T. B. Norris, "Adaptive correction of depth-induced aberrations in multiphoton scanning microscopy using a deformable mirror," J. Microsc. 206, 65-71 (2002).

[14] P. N. Marsh, D. Burns, and J. M. Girkin, "Practical implementation of adaptive optics in multiphoton microscopy," Opt. Express 11, 1123-1130 (2003).
[15] A. C. F. Conte and R. Dandliker, "Optimization of single-mode fiber coupling efficiency with an adaptive membrane mirror," Opt. Eng. 41, 1073-1076 (2002).

[16] A. J. Wright, D. Burns, B. A. Patterson, S. P. Poland, G. J. Valentine, and J. M. Girkin, "Exploration of the optimization algorithms used in the implementation of adaptive optics in confocal and multiphoton microscopy," Microsc. Res. Technol. 67, 36-44 (2005).

[17] M. J. Booth, "Wavefront sensor-less adaptive optics: a modelbased approach using sphere packings," Opt. Express 14, 1339-1352 (2006).

[18] M. Vorontsov, “Decoupled stochastic parallel gradient descent optimization for adaptive optics: integrated approach for wave-front sensor information fusion," J. 0pt. Soc. Am. A 19, 356-368 (2002).

[19] M. J. Booth, M. A. A. Neil, R. Juskaitis and T. Wilson, "Adaptive aberration correction in a confocal microscope," Proc. Nat. Acad. Sci. 99, 5788-5792 (2002).

[20] J. Antonello, M. Verhaegen, R. Fraanje, T. van Werkhoven, H. C. Gerritsen, and C. U. Keller, "Semidefinite programming for model-based sensorless adaptive optics," J. Opt. Soc. Am. A 29, 2428-2438 (2012).

[21] D. Debarre, M. J. Booth and T. Wilson, "Image-based adaptive optics for imaging and microscopy," Proc. SPIE 6888, 68880A (2008).

[22] M. Shaw, K. O'Holleran, K. Ryan, and C. Paterson, "Adaptive optics fluorescence microscopy of C. elegans," http://www.npl.co.uk/upload/pdf/ adaptive-optics-fluorescence-microscopy-of-c-elegans.pdf

[23] J. Garcia-Marquez, J. E. A. Landgrave, N. Alcala-Ochoa, and C. Perez-Santos, "Recursive wavefront aberration correction method for LCoS spatial light modulators," Opt. Laser Eng. 49, 743-748 (2011).

[24] J. M. Bueno, B. Vohnsen, L. Roso, and P. Artal, "Temporal wavefront stability of an ultrafast high-power laser beam," Appl. Optics 48, 770-777 (2009).

[25] J. Schwiegerling, "Scaling pseudo-Zernike expansion coefficients to different pupil sizes," Opt. Lett. 36, 3076-3078 (2011).

[26] J. Schwiegerling, "Description of Zernike Polynomials" www.visualopticslab.com/0PTI515L/Background/Zernike\% 20Notes\%2017Feb2011.pdf 